Original Contribution

\title{
OROPHARYNGEAL CANDIDIASIS AND ANTIFUNGAL ASSESSMENT OF CANDIDA GLABRATA IN PATIENTS WITH HIV INFECTION
}

\author{
A. Sharifzadeh ${ }^{1}$, H. Shokri ${ }^{*}$ \\ ${ }^{1}$ Mycology Research Center, Faculty of Veterinary Medicine, University of Tehran, Tehran, Iran \\ ${ }^{2}$ Faculty of Veterinary Medicine, Amol University of Special Modern Technologies, Amol, Iran
}

\begin{abstract}
The purposes of this study were to determine the prevalence of oral mycoflora in $\mathrm{HIV}^{+}$patients and to assay antifungal susceptibility profile of Candida glabrata (C. glabrata) isolates to standard antifungal agents. A total of 100 HIV-infected patients with oropharyngeal candidiasis (OPC) were selected in this study. Susceptibility of $C$. glabrata isolates to antifungals was determined using the disc diffusion method. A total of 254 yeasts were isolated from the patients. Forty percent $(40 \%)$ of the patients had angular cheilitis as the most frequent clinical variant. Candida species (94.4\%) were the most frequently obtained genera $(\mathrm{P}<0.05)$, followed by Saccharomyces $(2.4 \%)$, Kluyveromyces and Cryptococcus $(1.6 \%)$ species. C. albicans $(37.2 \%)$ was the most common species isolated from $\mathrm{HIV}^{+}$patients with OPC and its frequency was significantly higher than that of other Candida species $(\mathrm{P}<0.05)$. Among non-C. albicans species, $C$. glabrata was the most frequent species. Highest sensitivity of $C$. glabrata to antifungal drugs was seen against polyene drugs such as nystatin and amphotericin B. This study revealed huge diversity of Candida species and oral isolates of $C$. glabrata were most sensitive to polyene drugs such as nystatin and amphotericin B and least sensitive to fluconazole.
\end{abstract}

Key words: Oral cavity, candidiasis, Candida glabrata, HIV infection, nystatin, amphotericin B.

\section{INTRODUCTION}

Candida species are the most common opportunistic fungal pathogens in humans, with Candida albicans (C. albicans) being the most prevalent pathogen in mucosal and systemic fungal infections $(1,2)$. For decades, C. glabrata was considered as a non-pathogen, but recent reports suggest that the antibiotic resistance mechanisms and evolutionary pressure has led to the emergence of $C$. glabrata variants which are highly pathogenic and drug-resistant (3). Moreover, therapeutic strategies, like of immunosuppressive drug therapy, anti-mycotic therapies and the emergence of the acquired immunodeficiency syndrome (AIDS), have drastically increased C. glabrata infections (4). Indeed, depending on the site of infection, C. glabrata currently ranks as the second or third most frequently isolated Candida species from all reported

\footnotetext{
*Correspondence to: Dr. Hojjatollah Shokri, Faculty of Veterinary Medicine, Amol University of Special Modern Technologies, Imam Khomeini Street, $24^{\text {th }}$ aftab, Amol, Iran. Phone No.: +9821 61117151, Fax No.: +98 21 66933222, Email: hshokri@ausmt.ac.ir
}

cases of candidiasis $(5,6)$. Recently a shift has been noted from fungal disease caused by $C$. albicans to that of non-albicans species of Candida, such as glabrata, especially in HIV patients $(7,8)$. In addition, $C$. glabrataassociated oropharyngeal candidiasis infections in HIV patients tend to be more severe and more difficult to treat than infections due solely to $C$. albicans $(7,9)$. Despite the fact that oral Candida infections are not associated with mortality, they are a significant source of morbidity, and trigger chronic pain or discomfort upon mastication, which may limit nutrition intake in immunocompromised or elderly individuals (10).

Erroneous diagnosis and false-positive interpretations have rigorous impact on drug selection for antifungal chemotheraphy. Therefore, the selection of antifungal agents for treatment of $C$. glabrata infections can be somewhat problematic, especially in critically ill patients. In such cases, the availability of susceptibility testing results may facilitate therapeutic decision-making. This study aimed to determine the prevalence of oral mycoflora in $\mathrm{HIV}^{+}$patients and to assay antifungal 
susceptibility profile of $C$. glabrata isolates to fluconazole, ketoconazole, nystatin, clotrimazole, amphotericin B and flucytosine.

\section{MATERIALS AND METHODS \\ Patients}

The patients for this study were Iranian $\mathrm{HIV}^{+}$ men and women in Iranian Research Center for HIV/AIDS, Imam Khomeini Hospital, Tehran, Iran (IRCHA). They were outpatients who came to receive treatment. The HIV infection was confirmed by ELISA and western blot techniques. The patients were enrolled after providing informed verbal consent. A standardized data collection form was used for retrieving information such as sex, age, HAART, smoking, marriage, addiction, intravenous drug abuse, Hepatitis B, Hepatitis $\mathrm{C}$ and having dentures. Clinical information and data regarding any history of OPC or other opportunistic infection was received. Pregnant women and individuals under treatment with antifungals were excluded.

\section{Specimen collection}

Oral lesions were clinically diagnosed for each individual and the specimens were taken by clinician using sterile cotton stick swab from lesions, tongue and buccal mucosa.

Isolation and identification of yeasts Specimens for fungal evaluation obtained from the oral cavity were cultured on sabouraud's dextrose agar plates (Merck Co., Darmastdt, Germany) and CHROM agar TM Candida (Paris, France Company) directly. CHROM agar TM plates were cultured for primary diagnosis and differentiation of Candida isolates. A wet mount with $10 \% \mathrm{KOH}$ was used for microscopic examination of pseudohyphae and yeast cell forms. The sabouraud's dextrose agar plates were incubated aerobically at $30^{\circ} \mathrm{C}$ for 7 days. CHROM agar TM culture for identification of colony form and color, were incubated at $35^{\circ} \mathrm{C}$ for $72 \mathrm{~h}$. After incubation period, the yeasts were identified based on morphological features and growth parameters (11). Germ tube test was performed with fresh rabbit serum and fresh yeast colony and incubated at $37^{\circ} \mathrm{C}$ for $3 \mathrm{~h}$. For evaluation of Chlamydospore and filamentous forms production, isolates were cultured on Dalmau plates (cornmeal-Tween 80 agar) for $48 \mathrm{~h}$ at $30^{\circ} \mathrm{C}$ (12). The ability of the isolates to assimilate carbohydrate sources was determined with Rap IDTM yeast identification system (Remel, USA) according to the manufacture's instruction (13). Isolates identified as $C$. glabrata were submitted to molecular identification. The isolates of $C$. glabrata were analyzed by a polymerase chain reaction (PCR) procedure with a specific primer.

\section{DNA extraction}

Portion of suspicious colony were washed with PBS containing $0.5 \%$ SDS and $50 \mathrm{mM}$ EDTA, cells were disrupted with Freeze-Thawing method and glass beads, and centrifuged at $10000 \mathrm{~g}$ for $2 \mathrm{~min}$. Five hundred micro liter of lysis buffer were added to precipitated material and maintained at room temperature for 10 min. After adding $150 \mathrm{~mL}$ potassium acetate buffer $\mathrm{pH} 4.8(60 \mathrm{~mL}$ of $5 \mathrm{M}$ potassium acetate, $11.5 \mathrm{~mL}$ glacial acetic acid, $28.5 \mathrm{~mL}$ distilled water), the tube was vortexed briefly and cell debris and precipitated proteins were removed by centrifugation at $12000 \mathrm{~g}$ for 2 min. The supernatant was transferred to another Eppendorf tube and centrifuged as mentioned above. Then the supernatant was transferred to a new $1.5 \mathrm{~mL}$ Eppendorf tube, an equal volume of isopropyl alcohol was added. The tube was mixed briefly by inversion, centrifuged at $12000 \mathrm{~g}$ for $2 \mathrm{~min}$ and the supernatant was discarded. The resultant DNA pellet was washed three times in $300 \mathrm{~mL}$ of $70 \%$ (V/V) ethanol. After centrifuging at $12000 \mathrm{~g}$ for $1 \mathrm{~min}$, the supernatant was discarded. The DNA was dried and dissolved in $30 \mathrm{~mL}$ distilled water. In order to measure of concentration and purification of DNA, optical density (OD) was read and run in the agarose gel.

\section{PCR reaction}

All putative isolates of $C$. glabrata were final identified by PCR method. Two pairs of oligonucleotide primers were used in this method. The forward primer CGL1 (5'- TTA TCA CAC GAC TCG ACA CT- $3^{\prime}$ ) and rivers primer CGL2 (5' - CCC ACA TAC TGA TAT GGC CTA CAA-3') were specific for $C$. glabrata (GenBank accession nos. AB032177, AF167993) and amplified a 423 bp DNA fragment from 5.8S rDNA gene. For PCR with individual primer pairs, each reaction mixture contained $2 \mu \mathrm{L}(1 \mathrm{ng})$ of diluted genomic DNA template or $0.5 \mu \mathrm{L}$ of yeast cell suspension, 20 $\mathrm{mM}$ Tris- $\mathrm{HCl}$ (pH 8.4), $50 \mathrm{mM} \mathrm{KCl}, 1.5 \mathrm{mM}$ $\mathrm{MgCl}_{2}, \quad 0.2 \mathrm{mM}$ (each of the four) deoxyribonucleotide triphosphates, $0.5 \mu \mathrm{M}$ (each) primer, and $0.5 \mathrm{U}$ of Taq DNA polymerase (Cinacolon) in a totalvolume of 25 $\mu \mathrm{L}$. PCR amplification conditions were $5 \mathrm{~min}$ of denaturation at $95^{\circ} \mathrm{C}$, followed by 40 cycles of $95^{\circ} \mathrm{C}$ for $30 \mathrm{~s}, 58^{\circ} \mathrm{C}$ for $30 \mathrm{~s}$, and $72^{\circ} \mathrm{C}$ for $30 \mathrm{~s}$ and a final extension step of $72^{\circ} \mathrm{C}$ for 10 min (Techne TC512, England). A sample of 10 $\mu 1$ of product from each PCR was electrophoresed in a $1.5 \%$ agarose gel with 0.5 $\mu \mathrm{g}$ of ethidium bromide/ml and $1 \mathrm{X}$ Tris- 
acetate-EDTA buffer for 1 to $2 \mathrm{~h}$. DNA bands were visualized on a UV transilluminator.

\section{Antifungal assay}

All antifungal discs were obtained from Oxoid (Hampshire, UK). The determination and interpretation of anti- C. glabrata activity of the drugs were carried out by agar disc diffusion method, according to the protocol in M44-A for yeasts (14). Briefly, a suspension of C. glabrata $\left(0.1 \mathrm{ml}\right.$ of $10^{6}$ cell $\left./ \mathrm{ml}\right)$ was spread on sabouraud's dextrose agar plate. The filter paper discs of reference antifungals were placed on the inoculated plates. These plates, after standing at $4^{\circ} \mathrm{C}$ for $2 \mathrm{~h}$, were incubated at $37^{\circ} \mathrm{C}$ for $48 \mathrm{~h}$. The positive and negative controls were maintained with filter paper discs dipped in reference antifungals and sterile distilled water, respectively. The diameters of the inhibition zones were measured in millimeters $(\mathrm{mm})$. Antifungal assay was performed in duplicate. The test was performed with reference antifungals including fluconazole (25 $\mu \mathrm{g} / \mathrm{disc})$, ketoconazole (15 $\mu \mathrm{g} / \mathrm{disc})$, nystatin $(50 \mu \mathrm{g} / \mathrm{disc})$, clotrimazole $(30 \mu \mathrm{g} /$ disc $)$, amphotericin B (10 $\mu \mathrm{g} / \mathrm{disc})$ and flucytosine $(1 \mu \mathrm{g} / \mathrm{disc})$.

\section{Statistical analysis}

The statistical analysis was performed using Statistical Program for Social Science (SPSS) 11.0 for windows. Chi-Square test was used to determine differences in proportion of categorized variables. Continuous variables with an approximately normal distribution were tested using the Student's test. A $P$ value less than 0.05 was considered statistically significant.

\section{RESULTS}

A total of $100 \mathrm{HIV}^{+}$individuals with and without signs or symptoms of OPC were recruited into this study at Imam Khomeini Hospital, Tehran, Iran. The demographic characteristics of the study population were as follows. As illustrated in Table 1, all patients ranged in age from 5 to 72 years old, with a mean age of 32.3 years old. The majority of patients $(60 \%)$ were $31-50,23 \%$ in $11-30,15 \%$ in $>50$ and $2 \%$ in $0-10$ year's groups. Seventyeight subjects were male, and 22 were female. Fifty-six patients were married and 44 were unmarried.

Table 1. Demographic data of $100 \mathrm{HIV}^{+}$patients included in this study.

\begin{tabular}{|c|c|c|c|c|c|c|c|c|c|c|c|}
\hline & \multicolumn{2}{|c|}{$\operatorname{Sex}(\%)$} & \multicolumn{5}{|c|}{ Underlying factor $(\%)$} & \multicolumn{4}{|c|}{ Signs and symptoms (\%) } \\
\hline & Female & Male & Addiction & $\mathrm{AD}^{\dagger}$ & Smoking & $\mathrm{IDU}^{\ddagger}$ & Jail & $\mathrm{AC}^{*}$ & $\mathrm{EC}^{* *}$ & $\mathrm{HC}^{* * *}$ & $\mathrm{PC}^{* * * * *}$ \\
\hline \multicolumn{12}{|c|}{ Age (year) } \\
\hline 0-10 & 0 & 2 & 0 & 0 & 0 & 0 & 0 & 0 & 0 & 0 & 3 \\
\hline 11-30 & 6 & 17 & 12 & 10 & 14 & 7 & 5 & 21 & 24 & 12 & 23 \\
\hline 31-50 & 11 & 49 & 36 & 33 & 45 & 30 & 41 & 32 & 17 & 8 & 54 \\
\hline$>\mathbf{5 0}$ & 5 & 10 & 8 & 7 & 11 & 6 & 8 & 22 & 18 & 6 & 21 \\
\hline Total & 22 & 78 & 56 & 50 & 70 & 43 & 54 & 75 & 59 & 26 & 101 \\
\hline
\end{tabular}

$\left\lceil\right.$ AD: Artificial denture; $¥$ IDU: Intravenous drug user; ${ }^{*}$ AC: Angular cheilitis; ${ }^{* *}$ EC: Erythematose candidiasis ${ }^{*}$ HC: Hyperplastic candidiasis, ${ }^{* * * *}$ PC: Pseudomemberanous candidiasis

The patients had a history of smoking (70\%), HAART (65\%), addiction (56\%), jail (54\%), denture wearing $(50 \%)$ and intravenous drug user $(43 \%)$. Out of 100 patients presented a clinically detectable OPC, angular cheilitis $(40 \%)$ was the most frequent clinical variant, pseudomembranous candidiasis in $28 \%$ patients, thrush in $22 \%$ patients and erythematous and hyperplastic candidiasis in $5 \%$ patients (Table 1).

A total of 254 yeast colonies were isolated from the patients understudy, representing 4 different genera in the tested samples. Among these samples, Candida species (94.4\%) were the most frequently obtained genera isolated from all study participants $(\mathrm{P}<0.05)$, followed by Saccharomyces (2.4\%), Kluyveromyces and Cryptococcus (1.6\%). C. albicans (37.2\%) was the most common species isolated from $\mathrm{HIV}^{+}$ patients with OPC and its frequency was significantly higher than that of other Candida species $(\mathrm{P}<0.05)$. Of these patients, $C$. glabrata, C. dubliniensis, C. tropicalis, $C$. parapsilosis, C. krusei, C. lusitaniae, $C$. guilliermondii and $C$. norvegensis were also identified.

As shown in Figure 1, the RAPD banding pattern of different clinical isolates using the one specific primer for $C$. glabrata. Using the primer CGL, $C$. glabrata isolates yielded RAPD profiles with one strong band, with molecular size of $423 \mathrm{bp}$.

In this study, a total of $20 C$. glabrata isolates were identified and selected for antifungal susceptibility testing. Test results of the susceptibility to antifungal drugs were as 
SHARIFZADEH A., et al.

follows: ketoconazole: 17 isolates (85\%) susceptible, 2 (10\%) susceptible-dose dependent and 1 (5\%) resistant; amphotericin B: 20 isolates $(100 \%)$ susceptible; nystatin: 20 isolates (100\%) susceptible; clotrimazole: 12 isolates (60\%) susceptible, $8 \quad(40 \%)$ susceptible-dose dependent; flucytosine: 11 isolates (55\%) susceptible, 7 (35\%) susceptible-dose dependent and 2 (10\%) resistant; fluconazole: 11 isolates (55\%) susceptible, 2 (10\%) susceptible-dose dependent and 7 (35\%) resistant. The inhibition zones were ranged from 19 to 52 $\mathrm{mm}$ (mean value: $35.45 \mathrm{~mm}$ ) for ketoconazole, 18 to $32 \mathrm{~mm}$ (mean value: $23.3 \mathrm{~mm}$ ) for amphotericin B, 20 to $30 \mathrm{~mm}$ (mean value: $23.2 \mathrm{~mm}$ ) for nystatin, 15 to $35 \mathrm{~mm}$ (mean value: $21.45 \mathrm{~mm}$ ) for clotrimazole, 5 to $32 \mathrm{~mm}$ (mean value: $21.35 \mathrm{~mm}$ ) for flucytosine and 5 to $32 \mathrm{~mm}$ (mean value: $17.3 \mathrm{~mm}$ ) for fluconazole (Table 2). Regarding the data, it was revealed that nystatin and amphotericin $\mathrm{B}$ were the most effective antifungal drugs and fluconazole and flucytosine had the poorest activity.

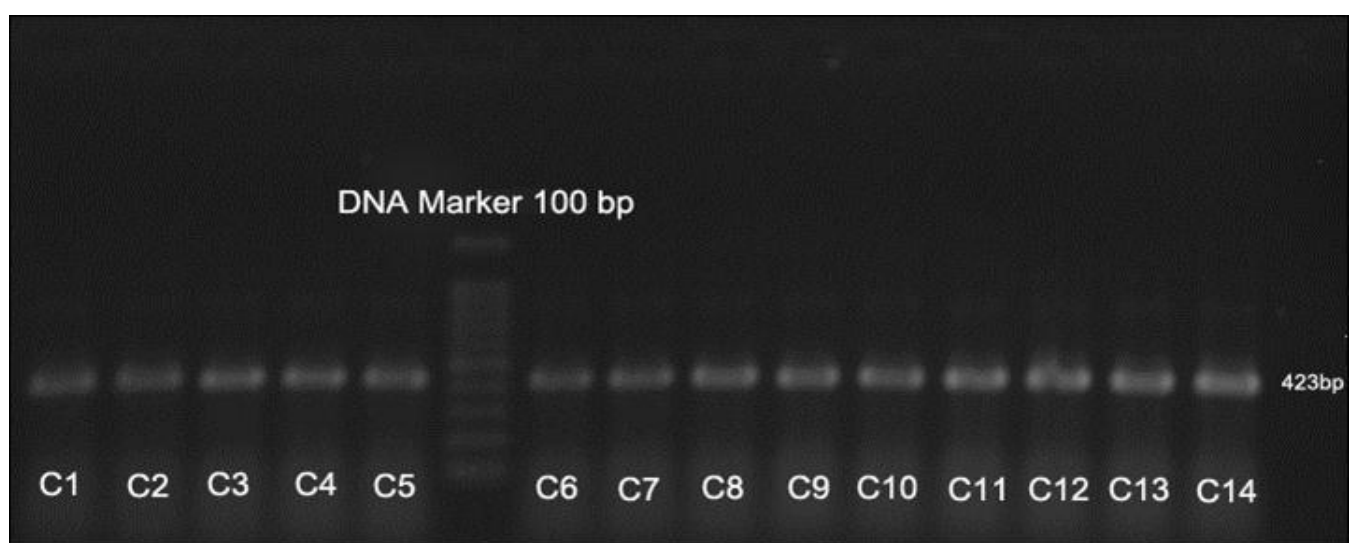

Figure 1. Primer pair designed to amplify DNA specifically from the Candida glabrata strains $\left(\mathrm{C}_{1}-\mathrm{C}_{14}\right)$ isolated from oral cavity of $\mathrm{HIV}^{+}$patients.

Table 2. Antifungal susceptibility of the reference antifungals against Candida glabrata isolates $\left(C_{1^{-}}\right.$ $\left.C_{20}\right)(\mathrm{mm})$.

\begin{tabular}{|c|c|c|c|c|c|c|}
\hline Isolate & Fluconazole & Ketoconazole & Clotrimazole & Nystatin & Amphotericin B & Flucytosine \\
\hline $\mathrm{C}_{1}$ & 32 & 52 & 30 & 26 & 27 & 19 \\
\hline $\mathrm{C}_{2}$ & 19 & 31 & 22 & 23 & 25 & 30 \\
\hline $\mathrm{C}_{3}$ & 20 & 37 & 20 & 20 & 21 & 30 \\
\hline $\mathrm{C}_{4}$ & 10 & 35 & 16 & 22 & 25 & 10 \\
\hline $\mathrm{C}_{5}$ & 7 & 34 & 15 & 20 & 20 & 14 \\
\hline $\mathrm{C}_{6}$ & 5 & 36 & 17 & 28 & 18 & 28 \\
\hline $\mathrm{C}_{7}$ & 20 & 22 & 16 & 25 & 22 & 30 \\
\hline $\mathrm{C}_{8}$ & 21 & 40 & 25 & 24 & 24 & 21 \\
\hline $\mathrm{C}_{9}^{\circ}$ & 31 & 48 & 35 & 24 & 25 & 13 \\
\hline $\mathrm{C}_{10}$ & 13 & 29 & 25 & 22 & 23 & 32 \\
\hline $\mathrm{C}_{11}$ & 20 & 33 & 15 & 23 & 23 & 5 \\
\hline $\mathrm{C}_{12}$ & 10 & 32 & 15 & 23 & 20 & 16 \\
\hline $\mathrm{C}_{13}$ & 21 & 43 & 31 & 30 & 32 & 18 \\
\hline $\mathrm{C}_{14}$ & 11 & 35 & 17 & 24 & 26 & 14 \\
\hline $\mathrm{C}_{15}$ & 21 & 39 & 22 & 24 & 24 & 19 \\
\hline $\mathrm{C}_{16}$ & 18 & 28 & 24 & 20 & 23 & 26 \\
\hline $\mathrm{C}_{17}$ & 7 & 19 & 22 & 21 & 22 & 27 \\
\hline $\mathrm{C}_{18}$ & 23 & 45 & 23 & 24 & 21 & 24 \\
\hline $\mathrm{C}_{19}$ & 19 & 27 & 22 & 21 & 25 & 31 \\
\hline $\mathrm{C}_{20}$ & 18 & 35 & 17 & 20 & 20 & 20 \\
\hline
\end{tabular}

\section{DISCUSSION}

The present study investigated $100 \mathrm{HIV}^{+}$ patients with OPC (one of the major markers of HIV disease progression) and included the $\mathrm{HIV}^{+}$groups regarding some important variables such as age, smoking, addiction and oral conditions (use of artificial dentures). The results showed that OPC in males (78\%) was more frequent than females $(22 \%)$, which were in accordance with other investigators $(15,16)$.
It seems that Iranian $\mathrm{HIV}^{+}$male patients have risk factors such as addiction, jail, intravenous drug user and smoking. These predisposing agents could also be important factors influencing the distribution frequency of Candida species among different age groups as well as the incidence of HIV infection (17). The most affected patients had 31-50 years old (60\%). Although different clinical signs of OPC appear in newborn and old ages, but in 
HIV patients because of decreasing $\mathrm{CD}_{4}{ }^{+}$ lymphocytes, every patient with HIV infection has a risk of the infection.

We also found a higher frequency of angular cheilitis $(40 \%)$ in $\mathrm{HIV}^{+}$patients regardless of underlying factors. In contrast, Katiraee et al. (18) observed thrush (38\%) as the most frequent clinical symptom in Iranian HIVinfected patients. In accordance with our findings, oral yeasts carriage, in particular Candida species has been demonstrated in asymptomatic $\mathrm{HIV}^{+}$patients (17), and an increased incidence of asymptomatic oral Candida carriage in $\mathrm{HIV}^{+}$patients compared to that in other at-risk groups has also been noted (18). Thus, a higher prevalence of oral $C$. albicans colonization may be a predisposing factor for the subsequent development of clinical candidiasis.

With respect to the Candida species identified in this study, C. albicans was isolated from $37.2 \%$ of $\mathrm{HIV}^{+}$patients. In accordance with our finding, the isolation of this species was reported by by Mousavi et al. (19), Katiraee et al. (16) and Shokohi et al. (17) in the oral cavity of $\mathrm{HIV}^{+}$patients. Even though $C$. albicans was the most common species recovered, the non-albicans Candida species (53.8\%) have become more recognized as a major source of infection. Recently, several reports have demonstrated a change in the oral Candida flora of HIV-infected patients. The use of various medicines such as antiretroviral agents, antibiotics and antifungal agents has been pointed as a reason for this change $(21,22)$. Among non-albicans Candida species, C. glabrata was the most frequently isolated species from the $\mathrm{HIV}^{+}$patients with OPC. In a study conducted by Redding et al. (23) study, C. glabrata has emerged as a notable pathogenic agent in oral mucosa, either as a co-infecting agent with $C$. albicans or as the sole detectable species from oral lesions. In addition, C. glabrata-associated $\mathrm{OPC}$ in $\mathrm{HIV}^{+}$ patients tend to be more severe and more difficult to treat than infections due solely to $C$. albicans. The comparison of frequency of nonalbicans isolates in the present study with previous studies is complicated due to different inclusion and exclusion criteria and sampling methods (24).

C. glabrata is naturally resistant to a wide variety of pharmacological and host-derived molecules. The resistance of $C$. glabrata to antifungals continues to be a significant problem in fungal infections. In this study, the susceptibility of C. glabrata isolates to different chemical antifungals such as, fluconazole, ketoconazole, nystatin, clotrimazole, amphotericin B and flucytosine were compared. Highest sensitivity of $C$. glabrata to antifungal drugs was seen against polyene drugs such as, nystatin and amphotericin B (20 of 20), followed by ketoconazole (17 of 20) and clotrimazole (12 of 20). The resistance to fluconazole was observed in 7 isolates of $C$. glabrata. In test with flucytosine, the surprisingly wide inhibition zones obtained were probably due to the low molecular weight and high aqueous solubility of the molecule (25). Our results showed that nystatin and amphotericin B were the most effective antifungal drugs and fluconazole had the poorest activity. There are many studies indicating that fluconazole had less activity against Candida species $(26,27)$. Our data are in agreement with previous reports as well. In view of the resistance of $C$. glabrata to antifungal agents, it is interesting that this organism is innately resistant to azole antifungals, in particular fluconazole. Recent studies have demonstrated that, in addition to having innate resistance, $C$. glabrata can also acquire drug resistance and become more resistant after selection in the presence of fluconazole. One key mechanism used by $C$. glabrata to develop acquired resistance is to increase azole efflux from the yeast cell through overexpression of two ATP-binding cassette transporters, cdr1 and cdr2 (28). Moreover, $C$. glabrata can upregulate the expression of the CgERG11 gene, which encodes lanosterol 14- demethylase (29). Unlike the azole drugs that exert their effect by inhibition of fungal cytochrome P450 enzymes, the polyene antifungals such as, nystatin and amphotericin B, act by binding to ergosterol, in the fungal cell membrane. Resistance to the polyene antifungals remains an uncommon event among Candida isolates. The polyenes still have reliable activity against most of the Candida species, except $C$ lusitaniae, which is often intrinsically resistant $(30,31)$.

\section{CONCLUSIONS}

In summary, an investigation of $\mathrm{HIV}^{+}$patients allowed us to monitor the dynamics of fungal oral colonization with particular reference to Candida species and its evolution toward OPC and to evaluate the prognostic value of Candida carriage in the development of AIDS. Oral colonization and infection by yeasts in Iranian $\mathrm{HIV}^{+}$patients is composed of different species, including $C$. albicans and nonalbicans Candida species, in particular $C$. glabrata. Antifungal sensitivity testing revealed that oral isolates of $C$. glabrata were 
most sensitive to polyene drugs such as, nystatin and amphotericin $\mathrm{B}$, and least sensitive to fluconazole.

\section{ACKNOWLEDGMENTS}

This work was supported by the Research Council of the University of Tehran. The authors would like to thanks all patients who participated in this study.

\section{CONFLICT OF INTEREST}

No conflict of interest was declared by the authors.

\section{REFERENCES}

1. Pfaller, M. A.; Diekema, D. J.; Jones, R. N.; Messer, S. A.; Hollis, R., Trends in antifungal susceptibility of Candida spp. isolated from pediatric and adult patients with bloodstream infections: SENTRY Antimicrobial Surveillance Program, 1997 to 2000. Journal of Clinical Microbiology 40, 852-856, 2002.

2. Trick, W. E.; Fridkin, S. K.; Edwards, J. R.; Hajjeh, R. A.; Gaynes, R. P., National nosocomial infections surveillance system hospitals. Secular trend of hospital-acquired candidemia among intensive care unit patients in the United States during 19891999. Clinical Infectious Diseases 35, 627630, 2002.

3. Hitchcock, C. A.; Pye, G. W.; Troke, P. F.; Johnson, E. M.; Warnock, D. W., Fluconazole resistance in Candida glabrata. Antimicrobial Agents and Chemotherapy 37, 1962-1965, 1993.

4. Komshian, S. V.; Uwaydah, A. K.; Sobel, J. D.; Crane, L. R., Fungemia caused by Candida species and Torulopsis glabrata in the hospitalized patient: frequency, characteristics, and evaluation of factors influencing outcome. Reviews of Infectious Diseases 11, 379-390, 1989.

5. Fidel, P. L.; Vazquez, J. A.; Sobel, J. D., Candida glabrata: review of epidemiology, pathogenesis, and clinical disease with comparison to C. albicans. Clinical Microbiology Reviews 12, 80-96, 1999.

6. Pfaller, M. A., Nosocomial candidiasis: emerging species, reservoirs, and modes of transmission. Clinical Infectious Diseases 22, S89-S94, 1996.

7. Masia Canuto, M.; Gutierrez Rodero, F.; Ortiz de la Tabla Ducasse, V.; Hernandez Aguado, I.; Martin Gonzalez, C.; Sanchez Sevillano, A., Determinants for the development of oropharyngeal colonization or infection by fluconazole-resistant Candida strains in HIV-infected patients. European Journal of Clinical Microbiology and Infectious Diseases 19, 593-601, 2000.
8. Demiraslan, H.; Alabay, S.; Ulu-Kilic, A.; Borlu, M.; Doganay, M., Cutaneous candidiasis caused by Candida glabrata in a HIV/AIDS patient. International Journal of STD AIDS 24, 753-755, 2013.

9. Redding, S. W.; Kirkpatrick, W. R.; Dib, O.; Fothergill, A. W.; Rinaldi, M. G.; Patterson, T. F., The epidemiology of nonalbicans Candida in oropharyngeal candidiasis in HIV patients. Special Care in Dentistry 20, 178-181, 2000.

10.Olmos, M. A.; Araya, V.; Concetti, H.; Ramallo, J.; Piskorz, E.; Perez, H., Oesophageal candidiasis: clinical and mycological analysis. Acta Gastroenterologica Latinoamericana 35, 211-218, 2005.

11.Odds, F. C.; Davidson, A., Room temperature" use of CHROM agar Candida. Diagnostic Microbiology and Infectious Diseases 38, 147-150, 2000.

12.Kurtezman, C. P.; Fell, J. W., The Yeast, a taxonomic study. 4th ed., Amsterdam: Elsevier Science, 1988.

13.Sanguinetti, M.; Porta, R.; Sali, M.; La Sorda, M.; Pecorini, G.; Fadda, G., Evaluation of VITEK 2 and RapID Yeast Plus Systems for yeast species identification: experience at a large clinical microbiology laboratory. Journal of Clinical Microbiology 45, 1343-1346, 2007.

14.NCCLS., Reference method for antifungal disk diffusion susceptibility testing of yeasts; approved guideline. NCCLS document M44-A. Wayne: National Committee for Clinical Laboratory Standards, 2004.

15.Bravo, I.; Correnti, M.; Escalona, L.; Perrone, M.; Barito, A., Prevalence of oral lesions in HIV patients related to CD4 cell count and viral load in Venezuelan population. Medicina Oral, Patología Oraly Cirugía Bucal 11, E3339, 2006.

16.Katiraee, F.; Khosravi, A. R.; Khalaj, V.; Hajiabdolbaghi, M.; Khaksar, A.; Rasoolinejad, M., Oropharyngeal candidiasis and oral yeast colonization in Iranian Human Immunodeficiency virus positive patients. Journal De Mycolpgie Medicale 20, 8-14, 2010.

17.Pignato, S.; Salvo, S.; Coniglio, M. A.; Marranzano, M.; Faro, G.; Giammanco, G., Persistent oral and urinary Candida spp. carriage in Italian HIV-seropositive asymptomatic subjects. Journal of Preventive Medicine and Hygiene 50, 232235, 2009. 
18.Hoshi, N.; Mori, H.; Taguchi, H.; Taniguchi, M.; Aoki, H.; Sawada, T., Management of oral candidiasis in denture wearers. Journal of Prosthodontic Research 55, 48-52, 2011.

19.Mousavi, S. A.; Salari, S.; Rezaie, S.; Shahabi Nejad, N.; Hadizadeh, S.; Kamyabi, H., Identification of Candida species isolated from oral colonization in Iranian HIV-positive patients, by PCRRFLP method. Jundishapur Journal of Microbiology 5, 336-340, 2012.

20.Shokohi, T.; Hashemi Soteh, M.; Pouri, Z. S.; Hedayati, M.; Mayahi, S., Identification of Candida species using PCR-RFLP in cancer patients in Iran. Indian Journal of Medical Microbiology 28, 147, 2010.

21.Masiá Canuto, M. M.; Gutiérrez Rodero, F.; Ortiz de la Tabla Ducasse, V.; Martín González, C., Epidemiology of oropharyngeal colonization and infection due to non-Candida albicans species in HIV infected patients. Medical Clinics 112, 211-214, 1999.

22.Diz Dios, P.; Ocampo, A.; Otero, I.; Iglesias, I.; Martinez, C., Changes in oropharyngeal colonization and infection by Candida albicans in human immunodeficiency virus-infected patients. Journal of Infectious Diseases 183, 355356, 2001.

23.Redding, S. W, Dahiya, M. C.; Kirkpatrick, W. R.; Coco, B. J.; Patterson, T. F.; Fothergill, A. W., Candida glabrata is an emerging cause of oropharyngeal candidiasis in patients receiving radiation for head and neck cancer. Oral Surgery, Oral Medicine, Oral Pathology, Oral Radiology and Endodontology 97, 47-52, 2004.

24.Gonçalves, L. S.; Ferreira, S. M.; Silva, A.; Villoria, G. E.; Gostinha, L. H.; Colombo, A. P., Association of T CD4 lymphocyte levels and chronic perodontitis in HIVinfected Brazilian patients undergoing highly active anti-retroviral therapy: clinical results. Journal of Periodontology 76, 915922, 2005.

25.Favel, A.; Chastin, C.; Thomet, A. L.; Regli, P.; Michel-Nguyen, A.; Penaud, A., Evaluation of the $\mathrm{E}$ test for antifungal susceptibility testing of Candida glabrata. European Journal of Clinical Microbiology and Infectious Diseases 19, 146-148, 2000.

26. Million, L., Fluconazole-resistant recurrent oral candidiasis in human immunodeficiency virus positive patients: persistence of Candida albicans strains with the same genotype. Journal of Clinical Microbiology 32, 1115-1118, 1994.

27.Enwuru, C. A.; Ogunledun, A.; Idika, N.; Enwuru, N. V.; Ogbonna, F.; Aniedobe, M., Fluconazole resistant opportunistic oropharyngeal Candida and non-Candida yeast-like isolates from HIV infected patients attending ARV clinics in Lagos, Nigeria. African Health Sciences 8, 142148, 2008.

28.Sanglard, D.; Ischer, F.; Calabrese, D.; Majcherczyk, P. A.; Bille, J., The ATP binding cassette transporter gene CgCDR1 from Candida glabrata is involved in the resistance of clinical isolates to azole antifungal agents. Antimicrobial Agents and Chemotherapy 43, 2753-2765, 1999.

29.Miyazaki, H.; Miyazaki, Y.; Geber, A.; Parkinson, T.; Hitchcock, C.; Falconer, D. J., Fluconazole resistance associated with drug efflux and increased transcription of a drug transporter gene, PDH1, in Candida glabrata. Antimicrobial Agents and Chemotherapy 42, 1695-1701, 1998.

30. Vandeputte, P.; Tronchin, G.; Berge`s, T.; Hennequin, C.; Chabasse, D.; Bouchara, J. P., Reduced susceptibility to polyenes associated with a missense mutation in the ERG6 gene in a clinical isolate of Candida glabrata with pseudohyphal growth. Antimicrobial Agents and Chemotherapy 51, 982-990, 2007.

31.Ellis, D., Amphotericin B: spectrum and resistance. Journal of Antimicrobial Chemotherapy 49, 7-10, 2002. 